\title{
Effect of Magnetic Field on Unsteady Flow of second order Oldroyd Visco-Elastic Liquid between Two Parallel Oscillating Flat Plates
}

\author{
Anil Tripathi ${ }^{1}$, A. K. Sharma ${ }^{2}$ and K.K. Singh ${ }^{3}$ \\ ${ }^{1,2}$ Department of Mathematics, K.K. (P.G.) College, Etawah (U.P.), India. \\ ${ }^{3}$ Department of Mathematics, Agra College, Agra (U.P.), India.
}

\begin{abstract}
The aim of the present paper is to study the oscillatory motion of a conducting visco-elastic Oldroyd liquid of second order between two parallel flat plates under the influence of an uniform magnetic field applied perpendicularly to the flat plates. Both the plates are considered to be oscillating harmonically with different amplitudes and different frequencies. Some particular cases have also been discussed in detail.
\end{abstract}

Keywords: Magnetic field, MHD, Non-Newtonian fluid, Oldroyd fluid of second order, Visco-elastic fluid.

\section{Introduction}

The visco-elastic fluids are particular cases of non-Newtonian fluids which exhibit appreciable elastic behaviour and stress-strain velocity relations and are time dependent. The subject of Rheology is of great technological importance as in many branches of industry, the problem arises of designing apparatus to transport or to process substances which can not be governed by the classical stress-strain velocity relations. Examples of such substances and the process are many, the extrusion of plastics, in the manufacture of rayon, nylon or other textiles, fibres, visco-elastic effects are encountered when the spinning solutions and in the manufacture of lubricating greases and rubbers.

The magnetohydrodynamic flow means, the motion of the electrically conducting fluid in the presence of Maxwell electro-magnetic field. The flow of the conducting fluid is effectively changed by the presence of the magnetic field and the magnetic field is also perturbed due to the motion of the conducting fluid. This phenomenon is therefore interlocking in character and the discipline of this branch of science is called Magnetohydrodynamics and in short written as MHD. It is equally rich and admits wider applications in Engineering, Technology, Astrophysics and other applied sciences. It has tremendously developed in recent years and some of the monographs in this direction are due to Ferraro and Plumpton [1]; Pai [2]; Shercliff [3]; Sutton and Sherman [4]; Jefferey [5] and Cowling [6] etc.

Many researchers have paid their attention towards the application of visco-elastic fluid flow of different category under the influence of magnetic field through channels of various cross-sections such as Choubey [7]; Yadav and Singh [8]; Suverna and Venkataramana [9]; Krishna and Rao [10]; Kundu and Sengupta [11]; Ghosh and Ghosh [12]; Banerjee [13]; Tripathi, Sharma and Singh [14]; Bodosa [15]; Devika, Satya Narayana and Venkataramana [16]; Agrawal,Agrawal and Varshney [17]; Sengupta and Paul [18] etc.

In the present paper our aim is to discuss the oscillatory motion of a conducting visco-elastic Oldroyd liquid of second order between two parallel flat plates when the uniform magnetic field has been applied perpendicularly to the flat plates. Both the plates are considered to be oscillating harmonically with different amplitudes and different frequencies. Some particular cases have also been discussed in detail.

\section{Basic theory for second order Oldroyd visco-elastic liquid}

For slow motion, the rheological equations for second order Oldroyd visco-elastic liquid are: $\tau_{i j}=-p \delta_{i j}+\tau_{i j}^{\prime}$

$\left.\begin{array}{l}\left(1+\lambda_{1} \frac{\partial}{\partial t}+\lambda_{2} \frac{\partial^{2}}{\partial t^{2}}\right) \tau_{i j}^{\prime}=2 \mu\left(1+\mu_{1} \frac{\partial}{\partial t}+\mu_{2} \frac{\partial^{2}}{\partial t^{2}}\right) e_{i j} \\ e_{i j}=\frac{1}{2}\left(v_{i, j}+v_{j, i}\right)\end{array}\right\}$

where $\tau_{\mathrm{ij}}$ is the stress tensor, $\tau_{\mathrm{ij}}$ the deviatoric stress tensor, $\mathrm{e}_{\mathrm{ij}}$ the rate of strain tensor, $\mathrm{p}$ the fluid pressure, $\lambda_{1}$ the stress relaxation time parameter, $\mu_{1}$ the strain rate retardation time parameter, $\lambda_{2}$ the additional material constant, $\mu_{2}$ the additional material constant, $\delta_{\mathrm{ij}}$ the metric tensor, $\mu$ the coefficient of viscosity and $v_{\mathrm{i}}$ is the velocity components. 


\section{Formulation of the problem}

Let us consider, $\mathrm{d}$ be the distance between the parallel flat plates, $\mathrm{x}$-axis along the lower plate in the direction of flow of liquid and y-axis along the perpendicular to the plates. Suppose that the lower plate and upper plate execute oscillations with different amplitudes $v_{1}, v_{2}$ and different frequencies $\omega_{1}, \omega_{2}$ respectively.

Following the stress-strain relations, the equation of motion for visco-elastic Oldroyd liquid of second order between two oscillating flat plates under the influence of an uniform magnetic field applied perpendicularly to the plates, when induced magnetic effect is neglected, is given by

$$
\begin{gathered}
\left(1+\lambda_{1} \frac{\partial}{\partial \mathrm{t}}+\lambda_{2} \frac{\partial^{2}}{\partial \mathrm{t}^{2}}\right) \frac{\partial \mathrm{u}}{\partial \mathrm{t}}=-\frac{1}{\rho}\left(1+\lambda_{1} \frac{\partial}{\partial \mathrm{t}}+\lambda_{2} \frac{\partial^{2}}{\partial \mathrm{t}^{2}}\right) \frac{\partial \mathrm{p}}{\partial \mathrm{x}}+v\left(1+\mu_{1} \frac{\partial}{\partial \mathrm{t}}+\mu_{2} \frac{\partial^{2}}{\partial \mathrm{t}^{2}}\right) \frac{\partial^{2} u}{\partial y^{2}} \\
-\frac{\sigma \mathrm{B}_{0}^{2}}{\rho}\left(1+\lambda_{1} \frac{\partial}{\partial \mathrm{t}}+\lambda_{2} \frac{\partial^{2}}{\partial \mathrm{t}^{2}}\right) \mathrm{u}
\end{gathered}
$$

where $\mathrm{u}$ is the velocity of visco-elastic liquid in the direction of oscillation, $\mathrm{p}$ the fluid pressure, $\mathrm{t}$ the time, $v$ the kinetic viscosity, $\sigma$ the electrical conductivity and $B_{0}$ is the magnetic inductivity.

Assuming that the pressure gradient is zero, the eqn. (2) becomes

$\left(1+\lambda_{1} \frac{\partial}{\partial \mathrm{t}}+\lambda_{2} \frac{\partial^{2}}{\partial \mathrm{t}^{2}}\right) \frac{\partial \mathrm{u}}{\partial \mathrm{t}}=v\left(1+\mu_{1} \frac{\partial}{\partial \mathrm{t}}+\mu_{2} \frac{\partial^{2}}{\partial \mathrm{t}^{2}}\right) \frac{\partial^{2} \mathrm{u}}{\partial y^{2}}-\frac{\sigma \mathrm{B}_{0}^{2}}{\rho}\left(1+\lambda_{1} \frac{\partial}{\partial \mathrm{t}}+\lambda_{2} \frac{\partial^{2}}{\partial \mathrm{t}^{2}}\right) \mathrm{u}$

The boundary conditions are:

$\left.\begin{array}{l}u=v_{1} e^{-i \omega_{1} t}, \text { when } y=0 \\ u=v_{2} e^{-i \omega_{2} t}, \text { when } y=d\end{array}\right\}$

Introducing the following non-dimensional quantities:

$y^{*}=\frac{1}{d} y, u^{*}=\frac{d}{v} u, t^{*}=\frac{v}{d^{2}} t, \quad \omega_{1}^{*}=\frac{d^{2}}{v} \omega_{1}, \omega_{2}^{*}=\frac{d^{2}}{v} \omega_{2}$,

$\lambda_{1}^{*}=\frac{v}{d^{2}} \lambda_{1}, \quad \mu_{1}^{*}=\frac{v}{d^{2}} \mu_{1}, \lambda_{2}^{*}=\frac{v^{2}}{d^{4}} \lambda_{2}, \mu_{2}^{*}=\frac{v^{2}}{d^{4}} \mu_{2}$

in the eqns. (3) and (4), we get (after dropping the stars)

$\left(1+\lambda_{1} \frac{\partial}{\partial \mathrm{t}}+\lambda_{2} \frac{\partial^{2}}{\partial \mathrm{t}^{2}}\right) \frac{\partial \mathrm{u}}{\partial \mathrm{t}}=\left(1+\mu_{1} \frac{\partial}{\partial \mathrm{t}}+\mu_{2} \frac{\partial^{2}}{\partial \mathrm{t}^{2}}\right) \frac{\partial^{2} \mathrm{u}}{\partial y^{2}}-\mathrm{H}\left(1+\lambda_{1} \frac{\partial}{\partial \mathrm{t}}+\lambda_{2} \frac{\partial^{2}}{\partial \mathrm{t}^{2}}\right) \mathrm{u}$

where $H=d B_{0} \sqrt{\frac{\sigma}{\mu}} \quad$ (Hartman number)

and boundary conditions are:

$\left.\begin{array}{l}u=v_{1} e^{-i \omega_{1} t}, \text { when } y=0 \\ u=v_{2} e^{-i \omega_{2} t}, \text { when } y=1\end{array}\right\}$

For the solution of eqn. (5), we may take

\section{Solution of the Problem}

$u=v_{1} f(y) e^{-i \omega_{1} t}+v_{2} g(y) e^{-i \omega_{2} t}$

which is evidently periodic in $\mathrm{t}$

Substituting eqn. (7) in eqn. (5), we obtain

$v_{1} e^{-i \omega_{1} t}\left[\left(1-i \omega_{1} \mu_{1}-\omega_{1}{ }^{2} \mu_{2}\right) \frac{d^{2} f}{d y^{2}}+\left\{i \omega_{1}\left(1-i \omega_{1} \lambda_{1}-\omega_{1}{ }^{2} \lambda_{2}\right)-H\left(1-i \omega_{1} \lambda_{1}-\omega_{1}{ }^{2} \lambda_{2}\right)\right\} f(y)\right]$
$+v_{2} e^{-i \omega_{2} t}\left[\left(1-i \omega_{2} \mu_{1}-\omega_{2}{ }^{2} \mu_{2}\right) \frac{d^{2} g}{d y^{2}}+\left\{i \omega_{2}\left(1-i \omega_{2} \lambda_{1}-\omega_{2}{ }^{2} \lambda_{2}\right)-H\left(1-\omega_{2} \lambda_{1}-\omega_{2}{ }^{2} \lambda_{2}\right)\right\} g(y)\right]=0$ 
or

$$
\begin{aligned}
& v_{1} e^{-i \omega_{1} t}\left(1-i \omega_{1} \mu_{1}-\omega_{1}{ }^{2} \mu_{2}\right)\left[\frac{d^{2} f}{d y^{2}}+\frac{\left(1-i \omega_{1} \lambda_{1}-\omega_{1}{ }^{2} \lambda_{2}\right)\left(i \omega_{1}-H\right)}{\left(1-i \omega_{1} \mu_{1}-\omega_{1}{ }^{2} \mu_{2}\right)} f(y)\right]+ \\
& v_{2} e^{-i \omega_{2} t}\left(1-i \omega_{2} \mu_{1}-\omega_{2}{ }^{2} \mu_{2}\right)\left[\frac{d^{2} g}{d y^{2}}+\frac{\left(1-i \omega_{2} \lambda_{1}-\omega_{2}{ }^{2} \lambda_{2}\right)\left(i \omega_{2}-H\right)}{\left(1-i \omega_{2} \mu_{1}-\omega_{2}{ }^{2} \mu_{2}\right)} g(y)\right]=0
\end{aligned}
$$

or

$$
v_{1} e^{-i \omega_{1} t}\left(1-i \omega_{1} \mu_{1}-\omega_{1}{ }^{2} \mu_{2}\right)\left[\frac{d^{2} f}{d y^{2}}+m^{2} f\right]+v_{2} e^{-i \omega_{2} t}\left(1-i \omega_{2} \mu_{1}-\omega_{2}{ }^{2} \mu_{2}\right)\left[\frac{d^{2} g}{d y^{2}}+n^{2} g\right]
$$

where $m^{2}=\frac{\left(1-i \omega_{1} \lambda_{1}-\omega_{1}{ }^{2} \lambda_{2}\right)\left(i \omega_{1}-H\right)}{\left(1-i \omega_{1} \mu_{1}-\omega_{1}{ }^{2} \mu_{2}\right)}$

and $\quad n^{2}=\frac{\left(1-i \omega_{2} \lambda_{1}-\omega_{2}^{2} \lambda_{2}\right)\left(i \omega_{2}-H\right)}{\left(1-i \omega_{2} \mu_{1}-\omega_{2}^{2} \mu_{2}\right)}$

By assumption $v_{1}$ and $v_{2}$ can not be zero, therefore from eqn. (8), we have

$\frac{d^{2} f}{d y^{2}}+m^{2} f=0$

$\frac{d^{2} g}{d y^{2}}+n^{2} g=0$

From (6) and (7), the corresponding boundary conditions reduced to

$\left.\begin{array}{ll}f(y)=1, & g(y)=0, \quad \text { when } y=0 \\ f(y)=0, & g(y)=1, \quad \text { when } y=1\end{array}\right\}$

Solutions of eqns. (9) and (10) subject to boundary conditions (11) are given by

$$
f(y)=\frac{\sin m(1-y)}{\sin m}
$$

and $\quad g(y)=\frac{\sin n y}{\sin n}$

Now substituting the values of $f(y)$ and $g(y)$ in (7), we obtain the velocity of second order Oldroyd visco-elastic liquid between two oscillating flat parallel plates under the influence of an uniform magnetic field applied perpendicularly to the plates

$u=v_{1} \frac{\sin m(1-y)}{\sin m} e^{-i \omega_{1} t}+v_{2} \frac{\sin n y}{\sin n} e^{-i \omega_{2} t}$

\section{Particular Cases}

CASE I: If material constants $\lambda_{2}$ and $\mu_{2}$ both are zero i.e. $\lambda_{2}=0$ and $\mu_{2}=0$, then from (14), we get velocity of Oldroyd (1958) visco-elastic liquid between two oscillating flat plates under the influence of an uniform magnetic field

$u=v_{1} \frac{\sin m(1-y)}{\sin m} e^{-i \omega_{1} t}+v_{2} \frac{\sin n y}{\sin n} e^{-i \omega_{2} t}$

where $m=\left[\frac{\left(1-i \omega_{1} \lambda_{1}\right)\left(i \omega_{1}-H\right)}{\left(1-i \omega_{1} \mu_{1}\right)}\right]^{\frac{1}{2}}$

$$
n=\left[\frac{\left(1-i \omega_{2} \lambda_{1}\right)\left(i \omega_{2}-H\right)}{\left(1-i \omega_{2} \mu_{1}\right)}\right]^{\frac{1}{2}}
$$

CASE II: If both the flat plates oscillate with same amplitude and different frequencies i.e. $v_{1}=v_{2}=v($ say), then from (14), we get

$u=v\left[\frac{\sin m(1-y)}{\sin m} e^{-i \omega_{1} t}+\frac{\sin n y}{\sin n} e^{-i \omega_{2} t}\right]$

CASE III: If both the flat plates oscillate with same frequencies and different amplitudes i.e. $\omega_{1}=\omega_{2}=\omega$ (say), then from (14), we get 
$u=\left[v_{1} \frac{\sin \mathrm{m}(1-y)}{\sin \mathrm{m}}+v_{2} \frac{\sin n y}{\sin n}\right] e^{-i \omega t}$

where $\quad m=n=\left[\frac{\left(1-i \omega \lambda_{1}-\omega^{2} \lambda_{2}\right)(i \omega-H)}{\left(1-i \omega \mu_{1}-\omega^{2} \mu_{2}\right)}\right]^{\frac{1}{2}}$

CASE IV: If both the flat plates oscillate with same amplitude and same frequency

i.e. $v_{1}=v_{2}=v$ (say) and $\omega_{1}=\omega_{2}=\omega$ (say), then from (14), we get

$u=v\{(1-\cot m) \sin m y+\cos m y\} e^{-i \omega t}$

where $m=\left[\frac{\left(1-i \omega \lambda_{1}-\omega^{2} \lambda_{2}\right)(i \omega-H)}{\left(1-i \omega \mu_{1}-\omega^{2} \mu_{2}\right)}\right]^{\frac{1}{2}}$

CASE V: If magnetic field is withdrawn i.e. $\mathrm{H}=0$, then from (14), we get

$u=v_{1} \frac{\sin m(1-y)}{\sin m} e^{-i \omega_{1} t}+v_{2} \frac{\sin n y}{\sin n} e^{-i \omega_{2} t}$

where $\quad m=\left[\frac{i \omega_{1}\left(1-i \omega_{1} \lambda_{1}-\omega_{1}{ }^{2} \lambda_{2}\right)}{\left(1-i \omega_{1} \mu_{1}-\omega_{1}{ }^{2} \mu_{2}\right)}\right]^{\frac{1}{2}}$

$$
n=\left[\frac{i \omega_{2}\left(1-i \omega_{2} \lambda_{1}-\omega_{2}{ }^{2} \lambda_{2}\right)}{\left(1-i \omega_{2} \mu_{1}-\omega_{2}{ }^{2} \mu_{2}\right)}\right]^{\frac{1}{2}}
$$

\section{Conclusion}

The tendency of uniform magnetic field applied perpendicularly to the flat plates is to reduce the velocity of visco-elastic liquid of different category.

\section{References}

[1]. Ferraro,V. C. A. and Plumpton, C. (1966): Magneto fluid mechanics, Larendon press, Oxford, London

[2]. Pai, S. L. (1962): Magnetohydrodynamics and Plasma Dynamics, Prentice Hall London.

[3]. Shercliff, J. A. (1965): A Text Book of Magnetohydrodynamics, Pergamon, Press.

[4]. Sutton, G. W. and Sherman, A. (1965): Engineering Magnetohydrodynamics, Mc. Graw Hill Book Company.

[5]. Jefferey, A. (1966): Magnetohydrodynamics, Oliver and Boyd, Edinbergh and London, Newyark.

[6]. Cowling, T. G. (1976): Magnetohydrodynamics, Bristol, England Adam Higher Limited.

[7]. Choubey, K. R. (1985): Ind. Jour. Pure Appl. Math., Vol. 19 (8), p.-931.

[8]. Yadav, B. S. and Singh, K. K. (1990): Proc. Nat. Acad. Sci. India, Vol. 60 (A) No.1, p.-103.

[9]. Suverna, K. and Venkataramana, S. (2002): Acta Ciencia Indica, Vol. XXVIII M, No. 4, p.-485.

[10]. Krishna, D. V. and Rao P. N. (2005): Acta Ciencia Indica, Vol. XXXI M, No. 3, p.-917.

[11]. Kundu, S. K. and Sengupta, P. R. (2003): Bull. of Allahabad Mathematical Soc. Vol. 18, p.-73.

[12]. Ghosh, S. and Ghosh A. K. (2006): Ind. Jour. Pure Appl. Math., Vol. 36 (10), p.-529.

[13]. Banerjee, S. (2008): Ind. Jour. Theo. Phys., Vol 56 (1), p.-17.

[14]. Tripathi, A.; Sharma, A. K. and Singh, K. K. (2009): Ultra Scientist of Physical Sci. (USPS) Vol. 21 (3) M, p.-671.

[15]. Bodosa, G. (2012): Ind. Jour. Theo. Phys., Vol 60 (2), p.-119.

[16]. Devika, B.; SaytaNarayana, P. V, and Venkataramana, S. (2013): Int. Jour. Engg. Sci. Invention, Vol. 2, issue 2, p.-26.

[17]. Agrawal, V. P.; Agrawal, J. K. and Varshney, N. K. (2013): Int. Jour. Mathematical Archive (IJMA), Vol. 4 (11), p.-1.

[18]. Sengupta, P.R. and Paul, S. (2004): Ind. Jour. Theo. Phys., Vol. 52 (4), p.-327. 